\title{
Genome-wide identification of markers for selecting higher oil content in oil palm
}

\author{
Bin Bai ${ }^{1 \dagger}$, Le Wang ${ }^{1 \dagger}$, May Lee ${ }^{1}$, Yingjun Zhang ${ }^{1}$, Rahmadsyah², Yuzer Alfiko ${ }^{3}$, Bao Qing Ye ${ }^{1}$, Zi Yi Wan?', \\ Chin Huat Lim², Antonius Suwanto ${ }^{3,4}$, Nam-Hai Chua ${ }^{1,5}$ and Gen Hua Yue 1,6,7* $^{2}$
}

\begin{abstract}
Background: Oil palm (Elaeis guineensis, Jacq.) is the most important source of edible oil. The improvement of oil yield is currently slow in conventional breeding programs due to long generation intervals. Marker-assisted selection (MAS) has the potential to accelerate genetic improvement. To identify DNA markers associated with oil content traits for MAS, we performed quantitative trait loci (QTL) mapping using genotyping by sequencing (GBS) in a breeding population derived from a cross between Deli Dura and Ghana Pisifera, containing $153 \mathrm{~F}_{1}$ trees.

Results: We constructed a high-density linkage map containing 1357 SNPs and 123 microsatellites. The 16 linkage groups (LGs) spanned $1527 \mathrm{cM}$, with an average marker space of $1.03 \mathrm{cM}$. One significant and three suggestive QTL for oil to bunch (O/B) and oil to dry mesocarp (O/DM) were mapped on LG1, LG8, and LG10 in a $F_{1}$ breeding population, respectively. These QTL explained 7.6-13.3\% of phenotypic variance. DNA markers associated with oil content in these QTL were identified. Trees with beneficial genotypes at two QTL for O/B showed an average O/B of 30.97\%, significantly $(P<0.01)$ higher than that of trees without any beneficial QTL genotypes (average O/B of 28.24\%). QTL combinations showed that the higher the number of QTL with beneficial genotypes, the higher the resulting average $\mathrm{O} / \mathrm{B}$ in the breeding population.
\end{abstract}

Conclusions: A linkage map with 1480 DNA markers was constructed and used to identify QTL for oil content traits. Pyramiding the identified QTL with beneficial genotypes associated with oil content traits using DNA markers has the potential to accelerate genetic improvement for oil yield in the breeding population of oil palm.

Keywords: RAD-seq, Mapping, QTL, Palm oil content, Oil yield

\section{Background}

Oil palm (Elaeis guineensis, Jacq.), mainly cultivated in the equatorial tropics of Africa, Southeast Asia and South America, is the world's most efficient oilseed crop, as it produces about $25 \%$ of worldwide vegetable oil annually, 32\% of global oils and fats output in 2012 and $40 \%$ of worldwide edible vegetable oil in 2014, while accounting for just 5.5\% of global oil crop cultivation land use [1]. Palm oil is not only widely used in cooking and factories, but is also used for producing biofuels, which resulted in a continuous rise in demand for palm

\footnotetext{
* Correspondence: genhua@tll.org.sg

†Equal contributors

${ }^{1}$ Temasek Life Sciences Laboratory, 1 Research Link, National University of Singapore, Singapore 117604, Singapore

${ }^{6}$ Department of Biological Sciences, National University of Singapore,

Singapore 117543, Singapore

Full list of author information is available at the end of the article
}

oil in recent years [2]. Planting higher yielding palms derived from improved germplasm genotypes is the most economically effective approach to guarantee adequate yield results in oil palm production. Using the Tenera genotype with thin shell $(s h+, s h-)$, derived from crossing of Dura with thick shell $(s h+, s h+)$ and Pisifera with no shell (sh-, sh-), resulted in a $30 \%$ increase in oil yield per hectare, hence improving the crude palm oil (CPO) yield in conventional breeding from 2.0 to 4.1 tons/ha in the last 50 years [1]. Recently, the global average CPO yield is 3.5 tons/ha of oil, still much lower than the estimated theoretical potential at 18 tons/ha [1]. Hence, there is substantial potential to increase $\mathrm{CPO}$ yield [3]. Although considerable genetic improvements have been made in yield in oil palm in the last several decades, the conventional improvement of oil palm is a complicated process, primarily due to long breeding cycles (i.e. over 20 years for males and 10 years for females per cycle) [1]. It is 
also difficult to select for complex traits such as oil content and oil quality. Marker-assisted selection (MAS) and genomic selection (GS) are expected to accelerate genetic improvement in breeding programs $[4,5]$.

QTL mapping refers to the identification of the molecular markers associated with quantitative traits on the whole genome [6]. It is the essential step towards MAS [4]. DNA markers, evenly covering a genome, are essential for QTL mapping. With the advent of next generation sequencing (NGS), single nucleotide polymorphisms (SNPs) are attractive DNA markers to use due to their high abundance, uniform distribution and compatibility with automated genotyping platforms [7]. A large number of DNA markers are essential for constructing of highdensity genetic maps and fine mapping of QTL for important economical traits [7]. Restriction-site-associated DNA sequencing (RAD-seq) using NGS is an efficient approach to discover and genotype a large number of SNP markers $[8,9]$. It has resulted in rapid and cost-effective massive marker discovery and has been successfully used to construct high-density linkage maps and fine QTL mapping in several plant species $[10,11]$. In oil palm, several linkage maps have been constructed with simple sequence repeats (SSRs) markers and SNPs [12-16]. The genomes of Elaeis guineensis and another palm species Elaeis oleifera have been sequenced [17, 18], supplying the necessary reference genomes to facilitate genotyping by sequencing (GBS) [16]. In previous studies, QTL mapping has been conducted for shell thickness [12], bunch number (BN), fresh fruit bunch yield (FFB) and other yield traits $[12,13,19]$, oil yield, oil to bunch content $(\mathrm{O} / \mathrm{B})$, fatty acid composition [20-24], plant growth, sex ratio [25], and embryogenesis in oil palm [26]. Also, a genomewide association study (GWAS) identified three key loci for oil to dry mesocarp content (O/DM) in two palm populations of Deli $\times$ AVROS and Nigerian $\times$ AVROS in Malaysia [27]. However, the effects and locations of QTL identified for important traits in different palm populations are mostly different, suggesting that most QTL detected are population-specific. To facilitate MAS in oil palm, it is necessary to further identify and verify QTL for important traits in different genetic backgrounds.

Oil content traits (e.g. oil to bunch, oil to mesocarp, or oil to kernel) are important traits in oil palm production. Usually, oil yield is positively correlated with oil content in the fruits of oil palm [28]. The aim of this study was to construct a high-density linkage map and map QTL for $\mathrm{O} / \mathrm{B}$ and $\mathrm{O} / \mathrm{DM}$ in an oil palm breeding population generated from a cross of Deli Dura $\times$ Ghana Pisifera. The markers were identified using GBS. Using the markers closely linked with the significant QTL for oil content, the elite trees containing the QTL can be detected, which could accelerate improvement of oil yield in oil palm.

\section{Results}

\section{Variation of oil content traits in a breeding population of} Dura $\times$ Pisifera

The two oil content traits (i.e. O/B and O/DM) of the palm breeding population used for mapping QTL were evaluated for two and three harvest periods during 2011-2013, respectively. The average of the trait O/B was $30.18 \pm 0.21 \%$ whereas the average of the trait $\mathrm{O} / \mathrm{DM}$ was $79.23 \pm 0.23 \%$ (Additional file 1: Table S1). A significant difference between individuals was found in the two traits (Additional file 1: Table S1, Additional file 2: Table S2). The coefficients of variation (CV) across different years or periods were $8.6-11.4 \%$ for $\mathrm{O} / \mathrm{B}$ and $3.6-4.8 \%$ for O/DM (Additional file 1: Table S1). Statistical analysis of the phenotypic data showed that the two traits were normally distributed (Shapiro-Wilks test, $P>0.05$ ) (Additional file 3: Figure S1), which indicates polygenic variation.

\section{Genotyping SNPs and SSRs in a breeding population}

Five sequencing libraries containing $177 \mathrm{~F}_{1}$ trees from the population and two parents were constructed by double-digest RAD-seq approaches, and a total of 693.3 million clean reads were produced by the Illumina NextSeq 500 platform. After sequential quality filtering and sequence trimming, 20.7 and 17.7 million reads were produced for the two parents, and an average of 3.7 million clean reads were produced in each progeny. Using the data sets of the parental samples, a catalogue containing 80,113 loci was constructed. Then, the catalogue was used as reference for SNP discovery and genotyping in the mapping population using the program genotypes in Stacks software package [29]. After filtering out the markers with more than $20 \%$ missing data across all data sets, 2139 SNP markers were used for the mapping. From the mapping population, 24 out of 177 individuals were discarded for further analysis due to high missing data. All the SNP information of the remaining 153 palms, including map position and sequence, is shown in Additional file 4: Table S3. We also used a set of 150 microsatellites (SSRs) showing polymorphism between the two parental palms for genotyping the palm population. A total of 123 informative SSRs were used in the mapping population. The detailed information of SSRs, including map position and primer sequences, is shown in Additional file 4: Table S3. All the SNPs and SSRs were used to construct a high-density linkage map.

\section{Constructing a high-density linkage map and mapping the unassembled scaffolds of the genome in Elaeis guineensis}

To construct a high-density linkage map and finer map QTL for important commercial traits, we developed and 
genotyped SNP markers using GBS by an improved RAD-seq approach [30]. The linkage map comprising 18 groups correspond to 16 LGs of previously published linkage map [13] were constructed for the Tenera population, of these LGs, two were each split into two smaller subgroups (LG4a, LG4b, LG11a, and LG11b). The linkage map, shown in Fig. 1, contains 1357 SNPs and 123 microsatellites, spans a total distance of $1527 \mathrm{cM}$ and has an average marker space of $1.03 \mathrm{cM}$. The lengths of LGs ranged from 9.6 cM for LG9 to 221.5 for LG3, with an average of $95.4 \mathrm{cM}$. The marker intervals ranged from $0.46 \mathrm{cM}$ on LG9 to $2.1 \mathrm{cM}$ on LG5. In all LGs, except on LGs 3 and 5, all markers spaces were smaller than $20 \mathrm{cM}$. A summary of the markers, marker densities and genetic distances for each linkage group, and the relation of linkage groups corresponding to the draft genome [18] and the previous map [13], are shown in Table 1 and Additional file 4: Table S3.

To facilitate the improvement of the assembly of the oil palm genome [18], we tried to map unassembled scaffolds to our genetic linkage map. In our high-density linkage map, 1357 SNPs and 123 microsatellites were assigned into 16 chromosomes. The sequences flanking 1345 markers were successfully aligned to the reference Elaeis guineensis genome. A total of 115 unassembled scaffolds (Additional file 4: Table S4) were successfully assigned to the 16 corresponding chromosomes. We investigated the genome syntenic relationships between LGs and chromosomes (Fig. 2). A high level of genomic synteny was observed between each LG and its corresponding chromosome across the whole genome of Elaeis guineensis. 93.8\% of markers with known chromosome location were matched correctly from their linkage groups to the corresponding chromosomes (Fig. 2).

\section{Mapping QTL for oil content traits}

By QTL mapping, one significant QTL Qoil_bunch_1.1 for $\mathrm{O} / \mathrm{B}$, explaining up to $13.3 \%$ of phenotypic variance both in the first period and for averaged $\mathrm{O} / \mathrm{B}$ among the three periods, was detected on LG1. Furthermore, one suggestive QTL Qoil_bunch_8.1 for O/B on LG8 and two suggestive QTL (Qoil_mesocarp_8.1 and Qoil_mesocarp_10.1) for O/DM on LG8 and LG10 were also identified both in the first period and for averaged value among the multiple periods in this study, explaining 10.5, 13.0 and $7.6 \%$ of phenotypic variance, respectively (Fig. 3 and Table 2). The confidence intervals of the four QTL were $1.92,1.18,0.92$, and $0.14 \mathrm{cM}(P<0.05)$, respectively. The markers EgSNP49135 and EgSNP49181 flanked the significant QTL Qoil_bunch_1.1. The two genotype calls AT and TT of EgSNP49135 had average $\mathrm{O} / \mathrm{B}$ of 29.47 and $30.64 \%$, respectively, and the three genotypes CC, CT, and TT of EgSNP49181 had average $\mathrm{O} / \mathrm{B}$ of $30.74 \%, 30.54 \%, 29.56 \%$, respectively (Additional file 5: Table S5), hence showing significant differences in $\mathrm{O} / \mathrm{B}$ in the breeding population (Fig. $4, P<0.001$ ). The

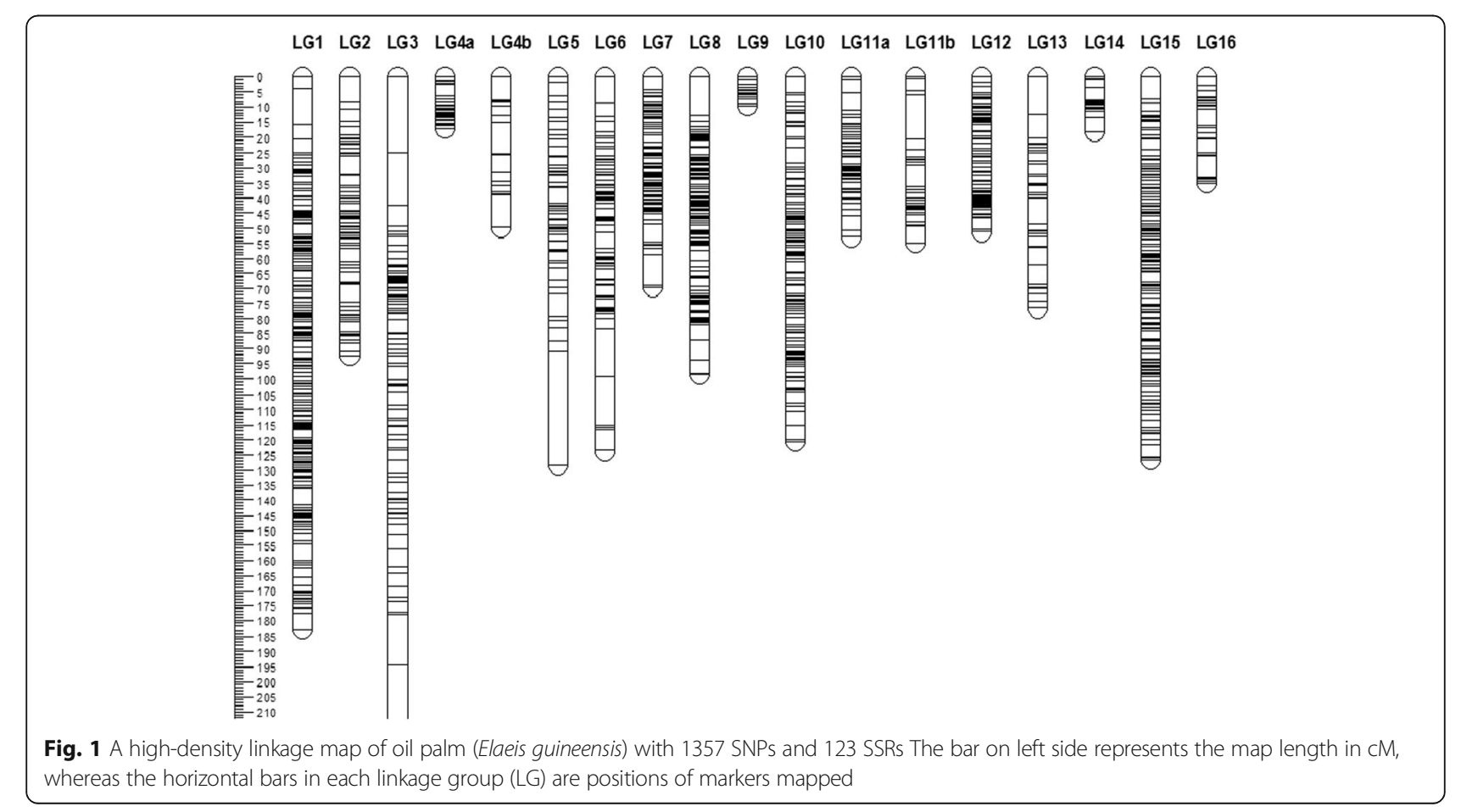


Table 1 Summary of statistics of the linkage map of oil palm Elaeis guineensis Jaca

\begin{tabular}{|c|c|c|c|c|c|c|}
\hline Linkage group & Markers & Length (cM) & Marker density (cM) & P2/Billotte ${ }^{a}$ & Chromosome/Singh $^{b}$ & Size(bp)/Singh ${ }^{b}$ \\
\hline LG 1 & 257 & 182.6 & 0.71 & LG 1 & CHR 3 & $60,058,032$ \\
\hline LG 2 & 76 & 92.3 & 1.21 & LG 2 & CHR 8 & $40,192,799$ \\
\hline LG 3 & 116 & 221.5 & 1.91 & LG 3 & CHR 14 & $24,378,543$ \\
\hline LG 4 & 49 & 66.5 & 1.36 & LG 4 & CHR 2 & $65,556,141$ \\
\hline LG 5 & 61 & 128.3 & 2.10 & LG 5 & CHR 16 & $21,370,583$ \\
\hline LG 6 & 85 & 123.4 & 1.45 & LG 6 & $\mathrm{CHR} 7$ & $43,453,266$ \\
\hline LG 7 & 95 & 69.4 & 0.73 & LG 7 & $\mathrm{CHR} 9$ & $38,054,796$ \\
\hline LG 8 & 154 & 98.3 & 0.64 & LG 8 & CHR 1 & $68,432,966$ \\
\hline LG 9 & 21 & 9.6 & 0.46 & LG 9 & CHR 13 & $27,816,170$ \\
\hline LG 10 & 139 & 120.5 & 0.87 & LG 10 & CHR 6 & $44,354,769$ \\
\hline LG 11 & 89 & 107.7 & 1.21 & LG 11 & $\mathrm{CHR} 4$ & $57,248,047$ \\
\hline LG 12 & 99 & 51.2 & 0.52 & LG 12 & CHR 5 & $51,953,839$ \\
\hline LG 13 & 39 & 76.5 & 1.96 & LG 13 & CHR 12 & $28,799,275$ \\
\hline LG 14 & 20 & 18.0 & 0.90 & LG 14 & CHR 11 & $30,067,610$ \\
\hline LG 15 & 154 & 126.4 & 0.82 & LG 15 & CHR 10 & $31,889,635$ \\
\hline LG 16 & 25 & 34.8 & 1.39 & LG 16 & CHR 15 & $24,313,565$ \\
\hline
\end{tabular}

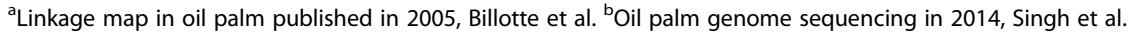

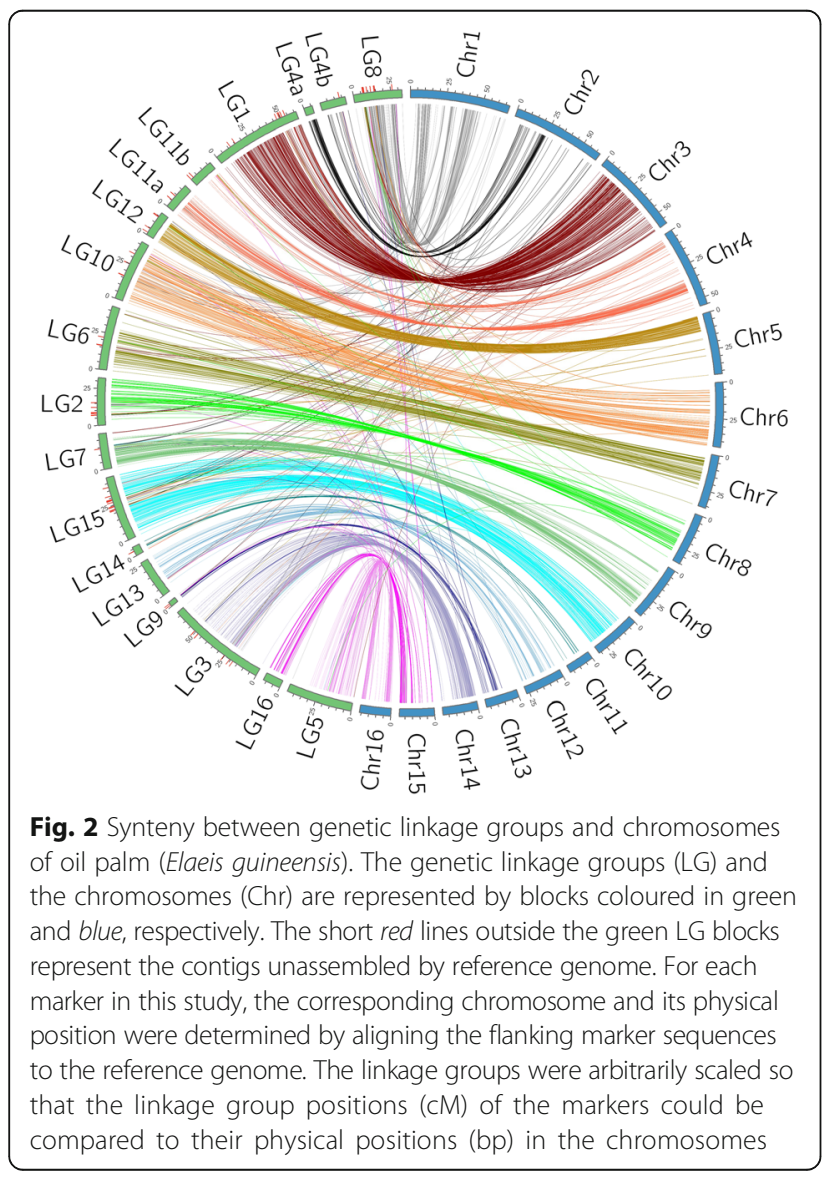

LOD value and the nearest SNPs to the peak of each QTL region are listed in Table 2.

The effects of QTL combinations on the increase of oil content

To elucidate the effect of different QTL combinations on the increase of oil content in the palm breeding population, the association with phenotypic data of marker genotypes in four QTL was analysed: Qoil_bunch_1.1, Qoil_bunch_8.1, Qoil_mesocarp_8.1, and Qoil_mesocarp_10.1. The average O/B of individual palms containing one or two O/B QTL with beneficial genotypes were 30.35 and $30.97 \%$, respectively, which was significantly higher than that of palms without those QTL with beneficial genotypes (average $\mathrm{O} / \mathrm{B}$ was $28.24 \%)(P<0.001)$. However, there was no significant difference between those having one and two O/B QTL with beneficial genotypes. The average $\mathrm{O} / \mathrm{B}$ of individuals containing three or four QTL with beneficial genotypes for both $\mathrm{O} / \mathrm{B}$ and $\mathrm{O} / \mathrm{DM}$ were 30.72 and $31.60 \%$, respectively. The average $\mathrm{O} / \mathrm{B}$ of individuals containing any one QTL with beneficial genotype was $29.34 \%$. The average $\mathrm{O} / \mathrm{B}$ of individuals containing three or four QTL with beneficial genotype was significantly higher than those individuals containing just one QTL with beneficial genotypes $(P<0.05)$, and the more QTL with beneficial genotype contained in the palm individuals, the higher the average $\mathrm{O} / \mathrm{B}$ (Fig. $5 \mathrm{a}$ ). On the other hand, the differences in average $\mathrm{O} / \mathrm{B}$ among palm individuals containing two to four QTL with beneficial genotype were not significant. 

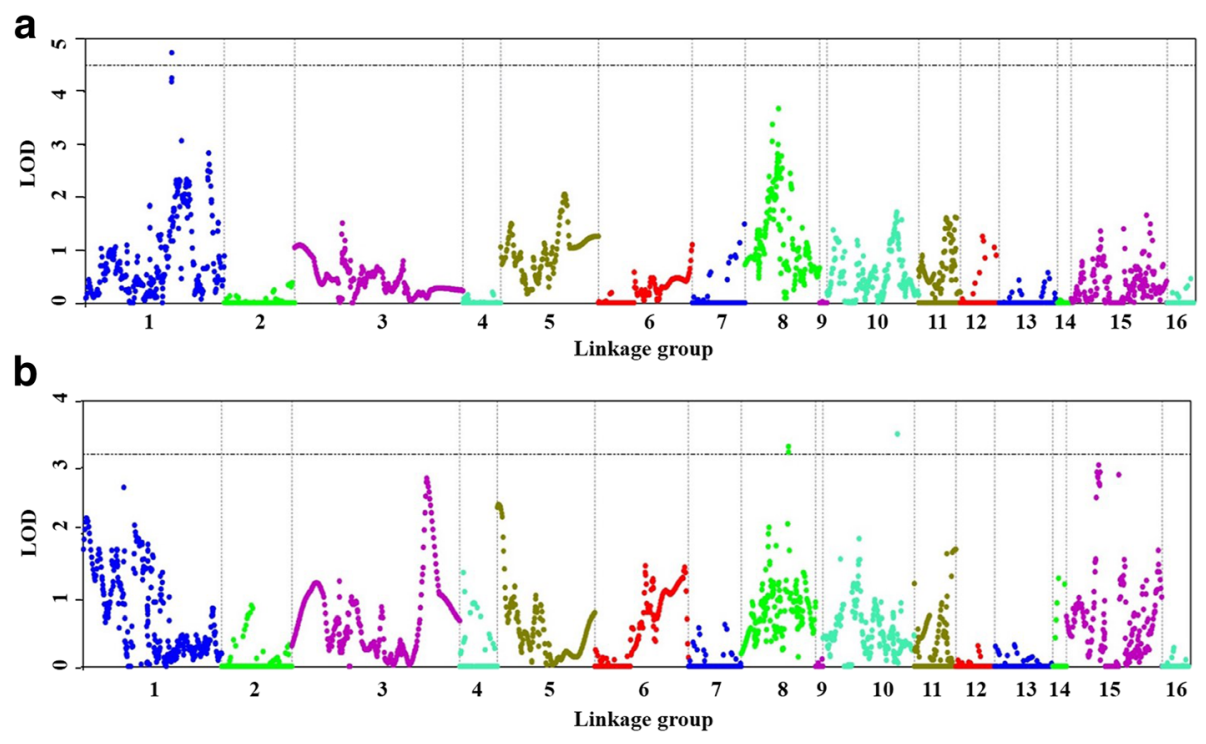

Fig. 3 Whole genome scan of QTL for oil content traits in oil palm (Elaeis guineensis Jacq.). a QTL for averaged oil to bunch content (O/B) from 3 years, b QTL for averaged oil to dry mesocarp content (O/DM) from 2 years. The dashed line shows genome-wide significance at $P=0.05$ (Figure a) and chromosome-wide significance at $P=0.05$ (Figure $\mathbf{b}$ )

As for $\mathrm{O} / \mathrm{DM}$, the average phenotypic values were 79.35, 78.59, and $79.67 \%$ among individuals containing beneficial genotypes of Qoil_mesocarp_8.1, Qoil_mesocarp_10.1, and both Qoil_mesocarp_8.1 and Qoil_mesocarp_10.1, respectively. These three groups had no significant difference between one another, but all had almost significant higher average O/DM values than those without any O/DM QTL with beneficial genotype (Fig. 5b). Similarly, there was no significant difference in $\mathrm{O} / \mathrm{DM}$ among individuals containing two to four QTL with beneficial genotypes for both $\mathrm{O} / \mathrm{B}$ and $\mathrm{O} / \mathrm{DM}$ but these individuals all had higher average O/DM (79.14\%, 79.49\%, $79.12 \%$, respectively) than those containing just one QTL with beneficial genotype (78.92\%) (Fig. 5b).

\section{Discussion}

Genotyping by sequencing (GBS) for the heterozygous oil palm breeding population

With the rapid advancement in next generation sequencing technologies, together with cost decrease, GBS is now feasible for high-throughput SNP discovery and genotyping [8]. The ddRAD-seq with PstI-MspI used here for genotyping was an effective GBS method to get a large number of SNPs in plants [9, 31]. We used 48 bar codes and 12 index (adapter) sets for the Illumina NextSeq 500 platform, which produced paired-end raw reads of $2 \times 150 \mathrm{bp}$ in length. The GBS approach has been used firstly in a $F_{2}$ population of Asian seabass for genotyping, which produced more than 18,000 SNPs across all the samples, and finally 3928 SNPs were

Table 2 Summary of QTL for oil content traits identified in the palm population of Dura $\times$ Pisifera

\begin{tabular}{|c|c|c|c|c|c|c|c|}
\hline Trait & QTL & Linkage group & QTL interval ${ }^{\mathrm{a}}(\mathrm{cM})$ & LOD Threshold $^{b}$ & LOD at peak & $\mathrm{PVE}^{\mathrm{C}}(\%)$ & $\begin{array}{l}\text { The nearest linkage } \\
\text { marker }^{d}\end{array}$ \\
\hline \multirow[t]{2}{*}{ oil to bunch_1st period } & Qoil_bunch_1.1 & LG1 & $113.1-115.0$ & 4.0 & 4.6 & 13.0 & EgSNP49153***** \\
\hline & Qoil_bunch_8.1 & LG8 & $44.1-45.3$ & 3.3 & 2.7 & 7.9 & EgSNP61696** \\
\hline \multirow[t]{2}{*}{ oil to bunch_average } & Qoil_bunch_1.1 & LG1 & $113.1-115.0$ & 3.8 & 4.7 & 13.3 & EgSNP49153*** \\
\hline & Qoil_bunch_8.1 & LG8 & $44.1-45.3$ & 3.4 & 3.7 & 10.5 & EgSNP61696****** \\
\hline \multirow{2}{*}{$\begin{array}{l}\text { oil to dry mesocarp_1st } \\
\text { period }\end{array}$} & Qoil_mesocarp_10.1 & LG10 & $98.1-99.0$ & 3.5 & 3.5 & 10.0 & EgSNP40659* \\
\hline & Qoil_mesocarp_8.1 & LG8 & $62.5-62.6$ & 3.2 & 3.3 & 9.5 & Eg0129* \\
\hline \multirow[t]{2}{*}{ oil to dry mesocarp_average } & Qoil_mesocarp_10.1 & LG10 & $98.1-99.0$ & 3.5 & 4.6 & 13.0 & EgSNP40659* \\
\hline & Qoil_mesocarp_8.1 & LG8 & $62.5-62.6$ & 3.4 & 3.4 & 7.6 & Eg0129* \\
\hline
\end{tabular}

${ }^{\mathrm{a}}$ significant confidence intervals $(P<0.05),{ }^{\mathrm{b}}$ chromosome-wide LOD threshold, ${ }^{\mathrm{c}}$ percentage of the phenotypic variance explained at the QTL, ${ }^{\mathrm{d}}$ significance from Kruskal-wallis analysis by software MapQTL6

${ }^{*}: P<0.05,{ }^{* *}: P<0.01,{ }^{* * *}: P<0.005,{ }^{* * * *}: P<0.001,{ }^{* * * * *}: P<0.0005,{ }^{* * * * * *}: P<0.0001$ 

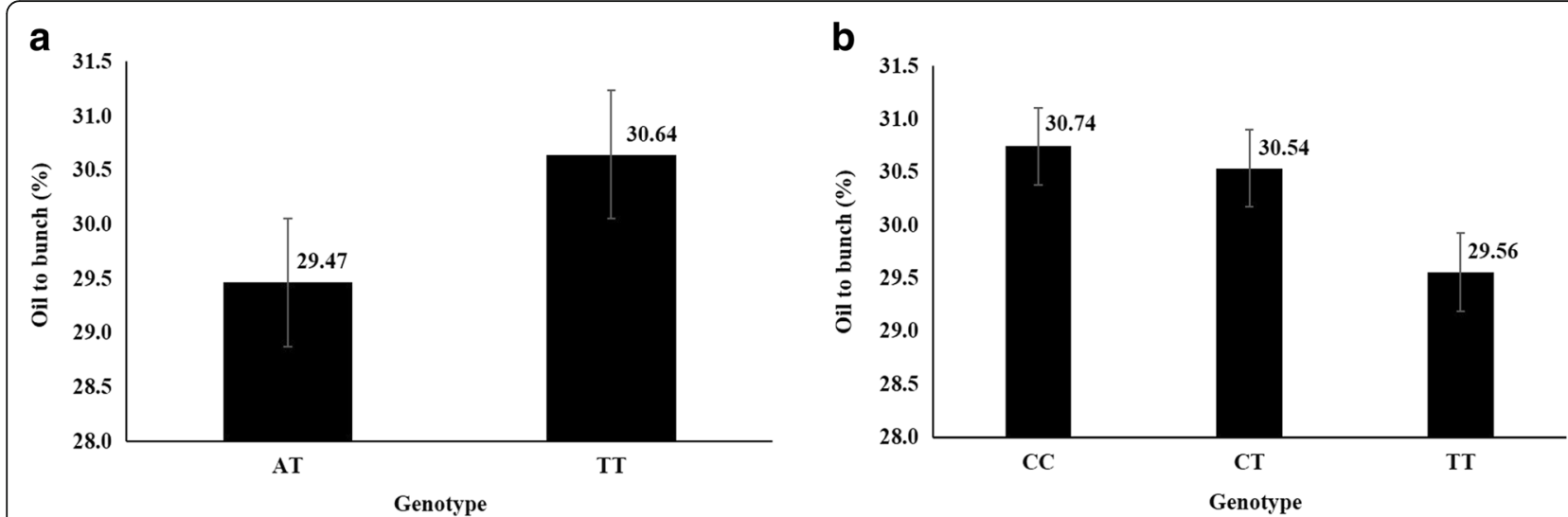

Fig. 4 Differences of trait values between different genotypes at two SNPs flanking the QTL for average O/B in an oil palm breeding population a Average O/B at two different genotypes of marker EgSNP49153 from QTL region on LG1 b Average O/B at two different genotypes of marker EgSNP49181 from QTL region on LG1

genotyped in more than $80 \%$ of progenies [30]. Compared to the $\sim 700 \mathrm{Mb}$ Asian seabass genome, oil palm has a huge genome $\sim 1.8 \mathrm{~Gb}$ [18]. We genotyped a $\mathrm{F}_{1}$ breeding population of Tenera palms derived from crossing of Dura and Pisifera using the same GBS approach. Only 2139 SNPs were genotyped in more than $80 \%$ of the palm progenies. A similar low number of SNPs genotyped was reported in another study on GBS in an oil palm $\mathrm{F}_{2}$ population derived from crossing Dura and Pisifera [16]. The lower number of SNPs genotyped in oil palm may be because the Tenera individuals, being derived from crossing two different forms, have a high heterozygosity in the genome, resulting in a lower digest efficiency of endonuclease and hence less common
SNPs, than for crosses of the same forms or varieties. In the future, different endonucleases should be tested during the construction of ddRAD-seq libraries to improve the efficiency of GBS.

\section{The high-density linkage map and improved genomic assembly for unmapped scaffolds in Elaeis guineensis} In our study, we have constructed a high-density linkage map for oil palm, with 1480 DNA markers. The marker density was down to $1.0 \mathrm{cM}$ in the intraspecific genetic map, from the previous $1.26 \mathrm{cM}[16]$ and $1.4 \mathrm{cM}[14]$ for the Dura and Pisifera intraspecific integrated maps, respectively. In previous studies on linkage mapping in oil palm, linkage maps were constructed with 252 to 944
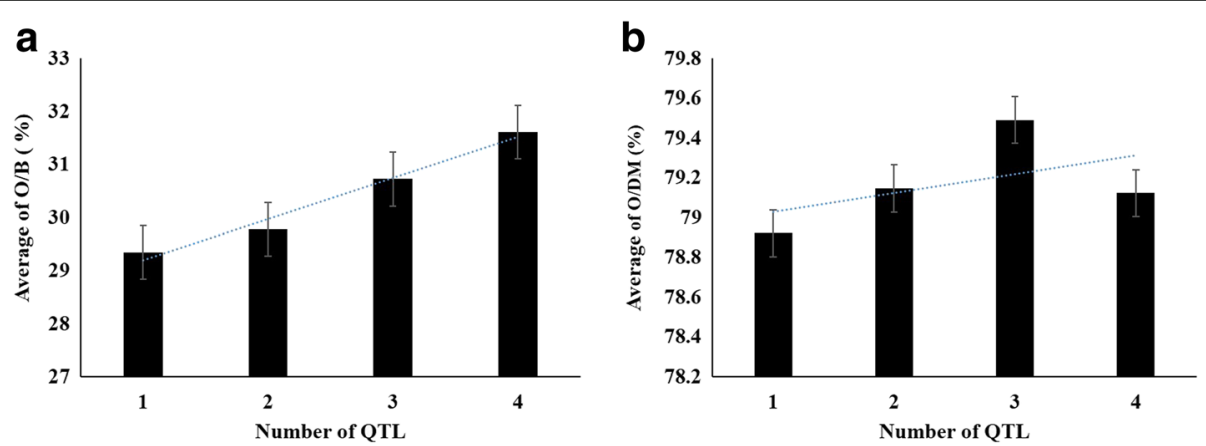

Fig. 5 Effect of the combinations of beneficial marker genotypes at four QTL for oil content on the increase of oil content in a Dura $\times$ Pisifera breeding population of oil palm (Elaeis guineensis Jacq.) a Effect of the combinations of QTL with beneficial marker genotypes on the increase of oil to bunch content (O/B). $\mathbf{b}$ Effect of the combinations of QTL with beneficial marker genotypes on the increase of oil to dry mesocarp content (O/DM). The numbers 1, 2, 3, and 4 represent number of the individuals containing the QTL number with beneficial genotypes. 1: individuals with one of QTL Qoil_bunch_1.1, Qoil_bunch_8.1, or Qoil_mesocarp_8.1, respectively. 2: individuals with QTL combinations Qoil_bunch_8.1 + Qoil_bunch_1.1, Qoil_mesocarp_10.1 + Qoil_bunch_1.1, Qoil_mesocarp_10.1 + Qoil_bunch_8.1, Qoil_mesocarp_8.1 + Qoil_bunch_1.1, Qoil_mesocarp_8.1 + Qoil_bunch_8.1, and Qoil_mesocarp_8.1 + Qoil_mesocarp_10.1, respectively. 3: individuals with QTL combinations Qoil_mesocarp_8.1 + Qoil_mesocarp_10.1 + Qoil_bunch_1.1, Qoil_mesocarp_8.1 + Qoil_mesocarp_10.1 + Qoil_bunch_8.1, Qoil_mesocarp_8.1 + Qoil_bunch_8.1 + Qoil_bunch_1.1, and Qoil_mesocarp_10.1 + Qoil_bunch_8.1 + Qoil_bunch_1.1, respectively. 4: individuals with QTLs combinations Qoil_bunch_8.1 + Qoil_bunch_1.1 + Qoil_mesocarp_8.1 + Qoil_mesocarp_10.1 
genetic markers, including RAPD, SSRs, and AFLPs [13-15, 20]. However, AFLP and RAPD markers are mainly dominant markers, and SSRs confer lower throughput and are more laborious to use than SNP. In addition, these previous linkage maps of oil palm had a limited marker number due to few numbers of markers available and small oil palm population. The development of $200 \mathrm{k}$ SNPs arrays and GBS technologies has enabled the genotyping of a large number of SNPs in larger oil palm populations, and thus the construction of high-density linkage maps $[9,27,31]$. In oil palm, a linkage map containing 1085 SNPs was constructed using GBS [16]. To the best of our knowledge, our linkage map is the densest linkage map of oil palm so far. The high-density linkage map will supply an essential tool for fine mapping QTL for MAS to accelerate genetic improvement of important traits. However, we noticed that on LGs 3 and 5, in a few positions, marker spaces were bigger than $20 \mathrm{cM}$, more markers should be mapped in these positions to reduce marker interval for QTL mapping. And two LGs (LG4, LG11) contained four small subgroups (LG4a, LG4b, LG11a, and LG11b) in this study, which could be due to the small number of markers called in the two LGs. One way to improve the marker density is to map the scaffolds of the sequenced genome [18] and re-sequenced genomes [17] to the linkage map using in-silico mapping [32]. Using our high-density genetic map, 115 unassembled scaffolds from the sequenced oil palm genome [18] were successfully assigned to the 16 corresponding chromosomes (Additional file 4: Table S4), which improved the genome assembly of oil palm. Alignment of 1345 markers mapped in our linkage map to the reference genome revealed a high level of genomic synteny between each LG and its corresponding chromosome across the whole genome of Elaeis guineensis. However, we noticed that $6.2 \%$ of aligned segments located on 16 chromosomes were not syntenic into corresponding linkage groups. Some of these could be caused by recombination in the mapping population, and some could be caused by incorrect genotyping or annotation in reference Pisifera genome sequence. Further improvement of the linkage maps by genotyping more DNA markers in larger mapping populations may improve the assembly of oil palm genome.

\section{QTL mapping for oil content related traits in Elaeis guineensis}

$\mathrm{O} / \mathrm{B}, \mathrm{O} / \mathrm{DM}$ and $\mathrm{FFB}$ yield are important components of oil yield in oil crops. Simultaneously increasing both traits is difficult to achieve in practical breeding programs because of the complex interdependence between plant developmental traits and yield components. On the other hand, oil content in fruit traits has generally high heritability in oil crops [33-35], and is also a complex quantitative trait that is directly related to oil yield in oil palm production [28]. We identified four QTL for $\mathrm{O} / \mathrm{B}$ and $\mathrm{O} / \mathrm{DM}$ averaged among all the periods in the breeding population. The four QTL were found on LG1, LG8, and LG10 in a Tenera population generated by crossing Deli Dura and Ghana Pisifera, respectively. Due to the variation of environments (different harvest periods), the phenotypic data of $\mathrm{O} / \mathrm{B}$ and $\mathrm{O} / \mathrm{DM}$ were significantly different in different harvest periods $(P<0.001$, Additional file 2: Table S2), thus a significant environmental effect on the population can be inferred. The four QTL mapped in the population were for one harvest period and mean $\mathrm{O} / \mathrm{B}$ and $\mathrm{O} / \mathrm{MD}$ from three and two harvest periods, respectively.

Only a few QTL for oil related traits had been identified in these LGs in previous studies [21, 27, 36]. There were some differences in location as well as phenotypic effect between our QTL and other previously reported QTL. In these known QTL, the QTL on LG1 for oil yield/palm/year, palm oil to pulp ratio [36], and O/DM [27], were different from Qoil_bunch_1.1 because of different traits and genomic location. The previous QTL for O/DM on LG10 was 21 Mbp away from Qoil_mesocarp_10.1 based on the physical position of the former's linkage marker mEgCIR3826 on the genome of oil palm [21], and the QTL for O/DM on LG10 was $\sim 27 \mathrm{M}$ bp away from Qoil_mesocarp_10.1 based on the oil palm genome [27]. Furthermore, we used the three key SNPs on chromosome 5 (corresponding to LG12) associated with O/DM detected by GWAS [27], to genotype two parental trees (Deli Dura and Ghana Pisifera) by Sanger sequencing in our mapping population. Unfortunately, two SNPs (TT genotype for SD_SNP_000010418, and CC genotype for SD_SNP_000002370) were not polymorphic in our mapping population, while the other (SD_SNP_000019529) were polymorphic, but not informative (i.e. TT genotype in Deli Dura and CC genotype in Ghana Pisifera). Therefore, in our population, we were not able to know the effects of these three SNPs. Compared to the previous studies on mapping of QTL for oil yield related traits in oil palm, the differences in the locations and effects of QTL for oil related traits suggest that the four QTL identified in this study could be novel loci and specific to our breeding population. All these data on QTL for oil content traits show that most QTL are populationspecific. This population-specificity of QTL could be caused by different germplasms being used in the mapping populations, different planation conditions or different numbers of markers used for the studies. More work should be conducted on the other elite palm germplasms, to fully detect the loci for oil yield related traits for MAS in oil palm improvement. 
DNA markers associated with oil content and QTL combinations for improving oil content

Many studies have shown that marker-based strategies of QTL pyramiding are effective and practicable in breeding programs $[37,38]$. Therefore, it is important to search for effective QTL associated with commercial traits. In our study, the genotypes with obvious negative and positive effects at four QTL associated with oil content traits were identified in an oil palm breeding population (Table 2 and Additional file 5: Table S5). Varying the combinations of genotypes of markers at these four QTL associated with $\mathrm{O} / \mathrm{B}$ or $\mathrm{O} / \mathrm{DM}$ showed that an increased number of QTL with positive effect, resulted in increased $\mathrm{O} / \mathrm{B}$ (Fig. 5a), and a slight increase for the $\mathrm{O} / \mathrm{DM}$ trait in the same breeding population (Fig. 5b). The obvious dosage effect showed that pyramiding these two O/B QTL and one or two of the O/DM QTL has great potential for oil palm breeding. Oil yield relative traits are complex and often controlled by large numbers of genetic loci with small additive effect [39], hence combining multiple QTL with beneficial genotypes in an individual can be an effective strategy of oil yield improvement in palm breeding.

Commercial seed production comes from crosses between phenotypically elite Dura and Pisifera trees, which produce higher yield Tenera trees for commercial plantation. MAS also can be used to identify the better Dura and Tenera individuals for the candidate of parents and tissue culturing, containing as many QTL as possible, in the early growth stage of the palms, instead of waiting till they are old enough for their yields to be measured. This reduces the generational interval and accelerates the genetic gain. Another way that MAS improves the efficiency of oil palm breeding is in the selection of Dura and parental Pisifera trees. The Pisifera, due to being female-sterile, and thus having no oil yield, were normally selected based on progeny tests, such as combining ability or heritability analysis by random choice, which need long, complicated selection cycles in palm breeding. MAS could be used to identify elite Pisifera trees carrying beneficial QTL, thus reducing the need for progeny tests. In our study, the beneficial genotypes of markers at QTL associated with oil content traits were identified in a breeding population. The latter was derived from a cross between an elite Deli Dura and an elite Ghana Pisifera, two parents which were used widely in the breeding program. Therefore, there is a great potential to pyramid these QTL in the breeding populations with similar genetic background as our mapping population. However, the effect of the QTL was based on statistical calculations in a particular breeding population, and the genetic background also has a great influence on the effect of QTL [40]. Hence, we need to verify the effectiveness of these QTL in other breeding populations with different genetic backgrounds from our population.

\section{Conclusions}

We constructed a high-density linkage map with 1480 DNA markers. This map will provide a basis for fine mapping of QTL and improve the assembly of the genome of Elaeis guineensis. Four QTL for oil content traits and DNA markers associated with these traits were identified in a breeding population. The QTL combinations have the potential to accelerate the improvement of oil content traits in our breeding population by MAS. Further verification of the detected QTL for oil content traits in other genetic backgrounds and plantation conditions is required to examine whether the QTL detected in this study can be used in MAS in other palm populations.

\section{Methods \\ Plant materials}

All plant materials were derived from a cross between an elite heterozygous Deli Dura tree and a heterozygous Ghana Pisifera tree from the oil palm breeding program of Wilmar International Plantation. A total of $177 \mathrm{~F}_{1}$ Tenera progenies were planted at a plantation field at the same time in 2006 and managed under the same conditions in Indonesia. The field arrangement of the populations was carried out following the standard protocol of Wilmar Plantation in Indonesia. This cross was used to assess the oil yield traits.

\section{Phenotypic traits recoding}

Oil to bunch content $(\mathrm{O} / \mathrm{B})$ was individually measured according to the standard protocol $[1,41]$ and recorded over three harvest periods (2011-2013). The oil to dry mesocarp content $(\mathrm{O} / \mathrm{DM})$ was measured according to the standard protocol $[1,41]$ and recorded for two harvest periods from six to seven years after planting (20122013). The oil content traits data from each year and arithmetic means for each individual were used for analysis of variance (ANOVA). The analysis of value distributions was performed with Excel 2013 (Microsoft).

\section{Genotyping microsatellites}

A total of 177 oil palm progenies and two parental samples were available for genotyping analysis. Leaf samples (the leaf tips) were collected from individual palms and stored at $-80{ }^{\circ} \mathrm{C}$ until DNA extraction. DNA was extracted and purified from leaf samples using the DNeasy Plant Mini kit (Qiagen, Germany). Genomic DNA quality was examined on $1.0 \%$ agarose gels, and quantification was conducted using Nanodrop 2000 (Nanodrop, DE, USA). 
Microsatellite markers were developed using an enrichment method described in our previous paper [15, 42]. A total of 150 microsatellite markers (Additional file 4: Table S3) were selected based on a published linkage map [15], distributed in a wide coverage within and across all the 16 linkage groups. The forward primers for each microsatellite were labelled with either 6-FAM or HEX fluorescent dye. PCR reaction for each sample consisted of $10 \mathrm{ng}$ of genomic DNA, 2.5 units of Taq-polymerase, $1 \times$ PCR buffer containing $1.5 \mathrm{mM}$ $\mathrm{MgCl}_{2}, 0.2 \mu \mathrm{M}$ dNTPs, and $50 \mathrm{nM}$ of each primer. PCR was performed on the thermal cycling machine (BioRad, CA, USA) under the following conditions: 3 min denaturation at $94{ }^{\circ} \mathrm{C}, 35$ cycles of $30 \mathrm{~s}$ at $94{ }^{\circ} \mathrm{C}$, $30 \mathrm{~s}$ at $52-58^{\circ} \mathrm{C}$ and $45 \mathrm{~s}$ at $72{ }^{\circ} \mathrm{C}$, and a final extension at $72{ }^{\circ} \mathrm{C}$ for $8 \mathrm{~min}$. Then the PCR products were analysed on an ABI3730x1 DNA sequencer (Applied Biosystems, Foster City, USA). The allele sizes were analysed against the ROX-500 standard (Applied Biosystems, Foster City, USA) using GeneMapper 4.1 (Applied Biosystems, Foster City, USA). Then the data were used for genotype analysis for parental palms and their $F_{1}$ palm progenies.

\section{RAD library preparation and sequencing}

A total of $177 \mathrm{~F}_{1}$ palm progenies derived from a cross between Dura and Pisifera, and the two parent trees, were used for constructing RAD libraries. The genomic DNA concentration for the RAD libraries was measured by Infinite ${ }^{\bullet}$ M1000 PRO plate reader (Tecan, Männedorf, Switzerland) using Qubit ${ }^{\bullet}$ dsDNA HS Assay Kit (Life Technologies, USA). RAD libraries were constructed using the double-digest RADseq method with some modifications [9] as described in the previous study [30]. A total of $200 \mathrm{ng}$ of genomic DNA from each sample was digested with 20 units of restriction enzymes PstI-HF and MspI (New England Biolabs, USA) at $37{ }^{\circ} \mathrm{C}$ for $3 \mathrm{~h}$. Then, the DNA fragments were examined by electrophoresis on a $2 \%$ agarose gel before ligation with barcoded adaptors [9]. The ligation products were pooled, followed by the size selection of 300-500 bp using Pipin Prep (Sage Science, USA), and then a clean-up using QIAquick PCR Purification Kit (Qiagen, Germany). The selected fragments of libraries were PCR amplified using Phusion ${ }^{\bullet}$ High-Fidelity DNA Polymerase (New England Biolabs, USA), followed by the second library clean-up using QIAquick PCR Purification Kit (Qiagen, Germany). Quantification of libraries was performed using KAPA Library Quantification Kits (Kapa Biosystems, USA) by qPCR in the MyiQ Thermal Cycler (Bio-Rad Laboratories, Hercules, CA, USA). Finally, the libraries were sequenced on a NextSeq 500 platform (Illumina, San Diego, CA, USA) to generate paired-end raw reads of $2 \times 150 \mathrm{bp}$ in length.

\section{SNP identifying and genotyping}

The raw reads were processed by the program process_radtags in Stacks package (version 1.21) [29] to remove low quality reads and any uncalled base, with a final step of de-multiplexing to assign clean reads to each sample. In order to reduce the sequencing errors at the end of each read, all the clean reads were trimmed to $125 \mathrm{bp}$. The cleaned reads were firstly aligned against the reference genome of oil palm [18] using the program BWAMEM algorithm with default parameters. The alignment products of each individual were further used for stacks assembly and catalogue construction using program pstacks $(-\mathrm{m}, 3)$ and cstacks in Stacks package. The assembled stacks of each sample were matched to a catalogue constructed using parental samples to discover SNPs using sstacks. The program genotypes was used to call SNPs across progeny. For SNP calling, a minimum of 20 times coverage was applied in two parents, and a minimum of five times sequence coverage was applied for each sample. Any discovered SNP with more than $20 \%$ missing data in both genotypes and individuals were removed from further analysis, which finally left 153 progeny of high level data quality for further analysis.

\section{Genetic linkage map construction, unassembled scaffolds mapping and QTL analysis}

The genotypic data of SSR and SNP markers for $153 \mathrm{~F}_{1}$ progeny palms derived from crossing Dura and Pisifera were used to construct genetic linkage maps with the software JoinMap 4.1 [43] and the genetic maps were drawn using the software MapChart V2.2 [44]. The markers with significant segregation distortion $(P<0.001)$ and markers having more than $10 \%$ missing data were excluded. Then the genotype data were grouped at LOD $\geq 4$. Next, 18 nodes were selected to create groups for calculating the genetic linkage groups. The linkage groups were calculated and markers ordered based on the regression mapping algorithm. The Kosambi mapping function was used to estimate the map distance. The linkage groups for the mapping population were numbered in correspondence to the map published based on the anchor markers and genomic sequence $[13,18]$.

The genome sequence of oil palm Elaeis guineensis [18], was used as reference sequence for SNP genotyping in our study. It was mostly assigned into the 16 chromosomes of oil palm, but part of the genomic scaffolds is still unmapped on the chromosomes. Sequence mapping of the RAD loci from the high-throughput genetic map were assigned into 16 chromosomes. Using this genetic map information, the unassembled scaffolds were assigned into the 16 corresponding chromosomes.

The QTL detection was performed using the software MapQTL 6 [45]. The Interval mapping (IM) method was performed with the algorithm of regression, mapping step 
size $1.0 \mathrm{cM}$, and a maximum number of five neighbouring markers. To declare the presence of a significant or suggestive QTL, the threshold LOD values were estimated at the genome-wide (GW), and chromosome wide levels (5\% of overall error level for both cases) by the permutation test (1000 permutations). The Kruskal-Wallis (K-W) test was performed to detect significant marker-trait associations at $P<0.05$. The confidence interval of each QTL was determined by the LOD threshold value in a permutation test $(P<0.05)$. The proportion of phenotypic variance explained (PVE) by a single QTL was calculated by the square of the partial correlation coefficient $\left(R^{2}\right)$ using the MapQTL software. The name for each QTL comprised of the trait, the linkage group, and a number to uniquely identify the QTL within the linkage group. For example, Qoil_bunch_1.1 represents the first QTL for oil to bunch located on linkage group LG1 in oil palm.

\section{Additional files}

Additional file 1: Table S1. Values of individual phenotypic traits, including means, ranges and coefficients of variation in the population of Dura $\times$ Pisifera. (DOCX $13 \mathrm{~kb})$

Additional file 2: Table S2. ANOVA for the phenotypic data of oil content traits in an oil palm breeding population of Dura $\times$ Pisifera. (DOCX $19 \mathrm{~kb}$ )

Additional file 3: Fig. S1. Distribution of phenotypic data (averaged over all periods) recorded in an oil palm breeding population used for QTL mapping. (JPEG $75 \mathrm{~kb}$ )

Additional file 4: Table S3. All the RAD markers and SSR markers with flanking sequences used in this study. Table S4. Mapping genome sequence of unknown chromosome in Elaeis guineensis, Jacq. (XLSX 182 kb)

Additional file 5: Table S5. Effects of marker genotypes at loci associated with oil content in a Dura $\times$ Pisifera breeding population. (DOCX 13 kb)

\section{Abbreviations \\ AFLP: Amplified fragment length polymorphisms; GBS: Genotyping by sequencing; GS: Genomic selection; GWAS: Genome-wide association study; LG: Linkage group; MAS: Marker-assisted selection; NGS: Next generation sequencing; O/B: Oil to bunch content (\%); O/DM: Oil to dry mesocarp content (\%); QTL: Quantitative trait loci; RAD-seq: Restriction-site-associated DNA sequencing; RAPD: Random amplified polymorphic DNA; SNP: Single nucleotide polymorphisms; SSR: Simple sequence repeats}

\section{Acknowledgements}

This research was supported by Wilmar International. We thank staff members of the R\&D department of Wilmar International Plantation for technical support.

\section{Funding}

This study was financially supported by Wilmar International and the internal fund of the Temasek Life Sciences Laboratory, Singapore.

\section{Availability of data and materials}

The RAD-seq datasets supporting the conclusions of this article are available in the DDBJ database, accession number (BioProject Accession: PRJDB4925).

\section{Authors' contributions}

NHC initiated and coordinated the project "Genetic Improvement of Oil Palm". NHC, GHY and BB designed the experiment and supervised the lab work. BB carried out the experiments and drafted the manuscript. LW contributed to the analysis related to the GBS analysis. ML prepared DNA samples and genotyping for SSR markers. R, YA, CHL and AS recorded traits and managed plants in field. BQY, ZYW and YJZ participated in genotyping GHY and NHC finalized the manuscript, and all authors read and approved the manuscript.

\section{Competing interests}

The authors BB, LW. YJZ, ML, BQY, ZYW, NHC and GHY declare that they have no competing interests. The authors $\mathrm{R}, \mathrm{YA}, \mathrm{JHL}$ and $\mathrm{AS}$ are employees of Wilmar International and Wilmar International funded this study.

Therefore, they have financial competing interests.

\section{Consent for publication}

Not applicable.

\section{Ethics approval and consent to participate}

All field studies were performed in accordance with local legislation in Indonesia and complied with the convention on the Trade in Endangered Species of Wild Fauna and Flora. We obtained a permission (i.e. a contract of research collaboration between Wilmar International and Temasek Life Sciences Lab) from Wilmar International to use its plant material and carry out our field study in its plantation field.

\section{Publisher's Note}

Springer Nature remains neutral with regard to jurisdictional claims in published maps and institutional affiliations.

\section{Author details}

${ }^{1}$ Temasek Life Sciences Laboratory, 1 Research Link, National University of Singapore, Singapore 117604, Singapore. ${ }^{2} \mathrm{R} \&$ D Department, Wilmar International Plantation, Palembang, Indonesia. ${ }^{3}$ Biotech Lab, Wilmar International, Jakarta, Indonesia. ${ }^{4}$ Bogor Agricultural University, Bogor, Indonesia. 'Laboratory of Plant Molecular Biology, The Rockefeller University, New York, USA. ${ }^{6}$ Department of Biological Sciences, National University of Singapore, Singapore 117543, Singapore. ${ }^{7}$ School of Biological Sciences, Nanyang Technological University, 6 Nanyang Drive, Singapore 637551, Singapore.

Received: 2 February 2017 Accepted: 22 May 2017

Published online: 30 May 2017

\section{References}

1. Corley RHV, Tinker PB. The oil palm. 5th ed. UK: Wiley; 2015. p. 1-26.

2. Kurnia JC, Jangam SV, Akhtar S, Sasmito AP, Mujumdar AS. Advances in biofuel production from oil palm and palm oil processing wastes: a review. Biofuel Res J. 2016:3(1):332-46.

3. Barcelos E, de Almeida RS, Cunha RN, Lopes R, Motoike SY, Babiychuk E, et al. Oil palm natural diversity and the potential for yield improvement. Front Plant Sci. 2015;6:190

4. Xu Y, Crouch JH. Marker-assisted selection in plant breeding: from publications to practice. Crop Sci. 2008;48(2):391-407.

5. Heffner EL, Sorrells ME, Jannink JL. Genomic selection for crop improvement. Crop Sci. 2009;49(1):1-12.

6. Geldermann H. Investigations on inheritance of quantitative characters in animals by gene markers I. Methods. Theor Appl Genet. 1975;46(7):319-30.

7. Zargar SM, Raatz B, Sonah H, Bhat JA, Dar ZA, Agrawal GK, et al. Recent advances in molecular marker techniques: insight into QTL mapping, GWAS and genomic selection in plants. J Crop Sci Biotechnol. 2015;18(5):293-308.

8. Elshire RJ, Glaubitz JC, Sun Q, Poland JA, Kawamoto K, Buckler ES, et al. A robust, simple genotyping-by-sequencing (GBS) approach for high diversity species. PLoS One. 2011;6(5):e19379.

9. Peterson BK, Weber JN, Kay EH, Fisher HS, Hoekstra HE. Double digest RADseq: an inexpensive method for de novo SNP discovery and genotyping in model and non-model species. PLoS One. 2012;7(5):e37135.

10. Bajgain P, Rouse MN, Anderson JA. Comparing genotyping-by-sequencing and single nucleotide polymorphism chip genotyping for quantitative trait loci mapping in wheat. Crop Sci. 2016;56(1):232-48.

11. Bielenberg DG, Rauh B, Fan S, Gasic K, Abbott AG, Reighard GL, et al. Genotyping by sequencing for SNP-based linkage map construction and QTL analysis of chilling requirement and bloom date in peach [Prunus persica (L.) Batsch]. PLoS One. 2015;10(10):e0139406. 
12. Moretzsohn MC, Nunes CDM, Ferreira ME. Grattapaglia D. RAPD linkage mapping of the shell thickness locus in oil palm (Elaeis guineensis Jacq.) Theor Appl Genet. 2000;100(1):63-70.

13. Billotte N, Marseillac N, Risterucci AM, Adon B, Brottier P, Baurens FC, et al. Microsatellite-based high density linkage map in oil palm (Elaeis guineensis Jacq.). Theor Appl Genet. 2005;110(4):754-65.

14. Ting NC, Jansen J, Mayes S, Massawe F, Sambanthamurthi R, Ooi LCL, et al. High density SNP and SSR-based genetic maps of two independent oil palm hybrids. BMC Genomics. 2014;15(1):309.

15. Lee $M$, Xia JH, Zou Z, Ye J, Alfiko Y, Jin J, et al. A consensus linkage map of oil palm and a major QTL for stem height. Sci Rep. 2015;5:8232.

16. Pootakham W, Jomchai N, Ruang-areerate P, Shearman JR, Sonthirod C, Sangsrakru D, et al. Genome-wide SNP discovery and identification of QTL associated with agronomic traits in oil palm using genotyping-bysequencing (GBS). Genomics. 2015;105(5):288-95.

17. Jin JJ, Lee M, Bai B, Sun YW, Qu J, Rahmadsyah, et al. Draft genome sequence of an elite Dura palm and whole-genome patterns of DNA variation in oil palm. DNA Res. 2016;23(6):527-33.

18. Singh R, Ong-Abdullah M, Low ETL, Manaf MAA, Rosli R, Nookiah R, et al. Oil palm genome sequence reveals divergence of interfertile species in old and new worlds. Nature. 2013;500(7462):335-9.

19. Rance K, Mayes S, Price Z, Jack P, Corley R. Quantitative trait loci for yield components in oil palm (Elaeis guineensis Jacq.). Theor Appl Genet. 2001 103(8):1302-10.

20. Singh R, Tan SG, Panandam JM, Rahman R, Ooi LC, Low ETL, et al. Mapping quantitative trait loci (QTLs) for fatty acid composition in an interspecific cross of oil palm. BMC Plant Biol. 2009;9(1):114

21. Jeennor S, Volkaert H. Mapping of quantitative trait loci (QTLS) for oil yield using SSRs and gene-based markers in African oil palm (Elaeis guineensis Jacq.). Tree Genet Genom. 2014;10(1):1-14.

22. Montoya C, Lopes R, Flori A, Cros D, Cuellar T, Summo M, et al. Quantitative trait loci (QTLs) analysis of palm oil fatty acid composition in an interspecific pseudo-backcross from Elaeis oleifera (H.B.K.) Cortés and oil palm (Elaeis guineensis Jacq.). Tree Genet Genom. 2013;9(5):1207-25.

23. Montoya C, Cochard B, Flori A, Cros D, Lopes R, Cuellar T, et al. Genetic architecture of palm oil fatty acid composition in cultivated oil palm (Elaeis guineensis Jacq.) compared to its wild relative E. oleifera (H.B.K) Cortés. PLoS One. 2014;9(5):e95412

24. King AJ, Montes LR, Clarke JG, Itzep J, Perez CA, Jongschaap RE, et al. Identification of QTL markers contributing to plant growth, oil yield and fatty acid composition in the oilseed crop Jatropha curcas L. Biotechnol Biofuels. 2015;8(1):160

25. Wong C, Bernardo R. Genomewide selection in oil palm: increasing selection gain per unit time and cost with small populations. Theor Appl Genet. 2008;116(6):815-24.

26. Ting NC, Jansen J, Nagappan J, Ishak Z, Chin CW, Tan SG, et al. Identification of QTLs associated with callogenesis and embryogenesis in oil palm using genetic linkage maps improved with SSR markers. PLoS One. 2013;8:e53076.

27. Teh CK, Ong AL, Kwong QB, Apparow S, Chew FT, Mayes S, et al. Genomewide association study identifies three key loci for high mesocarp oil content in perennial crop oil palm. Sci Rep. 2016;6:19075.

28. Okoye M, Okwuagwu C, Uguru M, Ataga C, Okolo E. Genotype by trait relations of oil yield in oil palm (Elaeis guineensis Jacq.) based on GT biplot. In: 8th African crop science Coference proceedings, vol. 6; 2007. p. 723-8.

29. Catchen JM, Amores A, Hohenlohe P, Cresko W, Postlethwait JH. Stacks: building and genotyping loci de novo from short-read sequences. G3 Genes Genom Genet 2011; 1(3):171-182.

30. Wang L, Wan ZY, Bai B, Huang SQ, Chua E, Lee M, et al. Construction of a high-density linkage map and fine mapping of QTL for growth in Asian seabass. Sci Rep. 2015;5:16358.

31. Sonah H, O'Donoughue L, Cober E, Rajcan I, Belzile F. Identification of loci governing eight agronomic traits using a GBS-GWAS approach and validation by QTL mapping in soya bean. Plant Biotech J. 2015;13(2):211-21.

32. Xia JH, Wan ZY, Ng ZL, Wang L, Fu GH, Lin G, et al. Genome-wide discovery and in silico mapping of gene-associated SNPs in Nile tilapia. Aquaculture. 2014:432:67-73.

33. Zhao J, Becker HC, Zhang D, Zhang Y, Ecke W. Conditional QTL mapping of oil content in rapeseed with respect to protein content and traits related to plant development and grain yield. Theor Appl Genet. 2006;113(1):33-8.
34. Kaushik N, Kumar K, Kumar S, Kaushik N, Roy S. Genetic variability and divergence studies in seed traits and oil content of Jatropha (Jatropha curcas L.) accessions. Biomass Bioenergy. 2007;31(7):497-502.

35. Fick G. Heritability of oil content in sunflowers. Crop Sci. 1975;15(1):77-8.

36. Billotte N, Jourjon M, Marseillac N, Berger A, Flori A, Asmady H, et al. QTL detection by multi-parent linkage mapping in oil palm (Elaeis guineensis Jacq.). Theor Appl Genet. 2010;120(8):1673-87.

37. Chen G, Habib A, Wei Y, Zheng YL, Shabala S, Zhou M, et al. Enhancing Fusarium crown rot resistance by pyramiding large-effect QTL in barley. Mol Breed. 2015;35(1):1-8.

38. Ortega MA, All JN, Boerma HR, Parrott WA. Pyramids of QTLs enhance host-plant resistance and Bt-mediated resistance to leaf-chewing insects in soybean. Theor Appl Genet. 2016;129(4):703-15.

39. Kainer D, Lanfear R, Foley WJ, Külheim C. Genomic approaches to selection in outcrossing perennials: focus on essential oil crops. Theor Appl Genet. 2015;128(12):2351-65.

40. Zhang B, Shi W, Li W, Chang X, Jing R. Efficacy of pyramiding elite alleles for dynamic development of plant height in common wheat. Mol Breed. 2013; 32(2):327-38.

41. Blaak G, Sparnaaij L, Menedez T. Breeding and inheritance in the oil palm (Elaeis guineensis Jacq.) II. Methods of bunch quality analysis. JW Afric Inst Oil Palm Res. 1963:4(14):146-55.

42. Yue $G$, Chen F, Orban L. Rapid isolation and characterization of microsatellites from the genome of Asian arowana (Scleropages formosus, Osteoglossidae, Pisces). Mol Ecol. 2000;9(7):1007-9.

43. Van Ooijen JW. JoinMap ${ }^{\oplus}$, software for the calculation of genetic linkage maps in experimental populations. Wageningen: Kyazma B V; 2006.

44. Voorrips RE. MapChart: software for the graphical presentation of linkage maps and QTLs. J Hered. 2002;93(1):77-8.

45. Van Ooijen JW. MapQTL ${ }^{\circledR} 6$. Software for the mapping of quantitative trait loci in experimental populations of diploid species. Wageningen: Kyazma BV; 2009.

\section{Submit your next manuscript to BioMed Central and we will help you at every step:}

- We accept pre-submission inquiries

- Our selector tool helps you to find the most relevant journal

- We provide round the clock customer support

- Convenient online submission

- Thorough peer review

- Inclusion in PubMed and all major indexing services

- Maximum visibility for your research

Submit your manuscript at www.biomedcentral.com/submit

) Biomed Central 